\title{
Flow and heat transfer analysis of Williamson nanofluid
}

\author{
S. Nadeem $\cdot$ S. T. Hussain
}

Received: 30 August 2013/ Accepted: 1 November 2013/Published online: 22 November 2013

(C) The Author(s) 2013. This article is published with open access at Springerlink.com

\begin{abstract}
In the present paper, we have examined the two-dimensional flow of Williamson fluid over a stretching sheet under the effects of nano-sized particle also described as nano Williamson fluid. The boundary layer equations of nano Williamson fluid model along with energy and nanoparticle volume fraction are presented and simplified with the help of useful transformations. Governing equations are somewhat different from the ones present in literature (reason is explained in the introduction section). The expressions for coefficients of skin friction and Nusselt number have been computed. The physical features of nondimensional Williamson parameter, Lewis number, Schmidt number and nano particle parameters (diffusivity ratio and heat capacities ratio) have been discussed by plotting the graphs of velocity, temperature and nanoparticle volume fraction.
\end{abstract}

Keywords Nano particles - Williamson fluid model · Stretching sheet $\cdot$ Heat transfer

\section{Introduction}

Nanofluids are colloidal suspension of base fluid and nanoparticles (1-100 nm). Nowadays these fluids are focus of research because these small size particles can enhance the coefficient of heat transfer several times as compared with base fluid. In some cases researchers have reported

\footnotetext{
S. Nadeem $\cdot$ S. T. Hussain $(\bowtie)$

Department of Mathematics, Quaid-I-Azam University 45320, Islamabad 44000, Pakistan

e-mail: s_thussain@yahoo.com

S. Nadeem

e-mail: snqau@hotmail.com
}

about $40 \%$ increase in thermal conductivity. This feature makes them very much suitable for cooling and solidification systems. In polymer industry solidification is an important phenomenon during polymers extrusion. Nanoparticles can play an important role in heat transfer during solidification. Also in extrusion of packaging films clay nanocomposites (Durmus and Kasgoz 2007) and silicate nanoparticles can be used, whose incorporation can create barrier for gases and increases the reliability of packaging films. Heat transfer enhancement due to these small size particles was first time reported by Masuda et al. (1993) This term was first introduced by Choi (1995); he defined nanofluid a liquid containing dispersed submicronic solid particles (nanoparticles). Three models have been widely used to describe the convective transport of nanofluids as discussed by Buongiorno (2006). As the first two models had drawbacks see Buongiorno (2006), he presented a third model and discussed several slip mechanisms and finally incorporated the effects of Brownian motion and thermophoretic diffusion into the equations. In the present article we will stick with Buongiorno model. Nield and Kuznetsov (2009) studied the thermal instability in a porous medium layer saturated by a nanofluid. They introduced the velocity similarity transformations dependent on thermal diffusivity of the porous medium. In another paper (Nield and Kuznetsov 2011) they considered the double diffusion: solute and nanoparticle diffusion in a base fluid. This time again, they introduced the velocity similarity transformations dependent on thermal diffusivity of fluid. Because of these similarity transformations Lewis number (Le) appears instead of Schmidt number $(S c)$ in their nanoparticle volume fraction equations. If we look in $\mathrm{Bu}-$ ongiorno's paper (Buongiorno 2006), in nanoparticle volume fraction equation, Schmidt number appears instead of Lewis number. Later on several authors (Makinde and Aziz 
2011; Khan and Pop 2010; Bachok et al. 2010; Kandasamya et al. 2011) citing the work of Nield and Kuzentsov introduced the Lewis number in nanoparticle volume fraction equation without considering the fact that their velocity transformations do not contain thermal diffusivity. These authors mistakenly reported the Schmidt number as Lewis number in their articles. If we do not choose velocity and stream functions dependent on thermal diffusivity then Lewis number will only appear in heat equation.

Stretching flows study has not lost its appeal yet due to its wide range of applications in polymer, glass, copper wire drawing and plastic industry. Sakiadis (1961) was the first one to study the boundary layer flow over a stretching sheet. Tsou et al. (1967) discussed the heat transfer effects on the boundary layer flow over a stretching sheet. Erickson et al. (1966) extended the work of Tsou et al. for mass transfer. Afterwards large numbers of theoretical studies have been carried out by numerous authors (Liu 2005; Rosali et al. 2012; Kelson and Farrell 2001; Kumaran and Ramanaiah 1996; Ali 1995).

Nadeem et al. (2013) were the first ones who developed the two-dimensional boundary layer equations for the flow of Williamson fluid past a stretching sheet. In the present article we are presenting the two-dimensional flow of nano Williamson fluid (Williamson 1929; Lyubimov and Perminov 2002; Nadeem 2010; Dapra 2007) over a stretching sheet. The governing boundary layer transport equations are first simplified using the suitable similarity transformations. Resulting equations are solved using the Homotopy analysis method (Liao 2003, 2004; Nadeem et al. 2010; Nadeem and Hussain 2009; Ellahi and Riaz 2010; Abbasbandy 2006, 2007; Hayat and Qasim 2010). To observe the convergence of obtained solution $h$-curve and convergence table are drawn. Graphs have been plotted to observe the impact of various physical parameters on transport phenomenon. Finally, tables are drawn to analyse the effects of important parameters on heat transfer coefficient and nanoparticle volume fraction gradient.

\section{Mathematical formulation}

Let us consider the two-dimensional steady flow of an incompressible nano Williamson fluid over a stretching surface. The plate is stretched along $x$-axis with a velocity $B x$, where $B>0$ is stretching parameter. The fluid velocity, temperature and nanoparticle concentration near surface are assumed to be $U_{\mathrm{w}}, T_{\mathrm{w}}$ and $C_{\mathrm{w}}$, respectively. The general transport equations for nanofluid are given by (Buongiorno 2006)

$\operatorname{div} \mathbf{V}=0$,

$\rho \frac{\mathrm{d} \mathbf{V}}{\mathrm{d} t}=\operatorname{div} \mathbf{S}+\rho \mathbf{b}$,

$$
\begin{aligned}
\rho c\left(\frac{\partial T}{\partial t}+\mathbf{V} \cdot \nabla T\right)= & \nabla \cdot k \nabla T+\rho_{\mathrm{p}} c_{\mathrm{p}} \\
& \times\left(D_{\mathrm{B}} \nabla \phi \cdot \nabla T+D_{\mathrm{T}} \frac{\nabla T \cdot \nabla T}{T_{\infty}}\right),
\end{aligned}
$$

$\frac{\partial C}{\partial t}+\mathbf{V} \cdot \nabla C=\nabla \cdot\left(D_{\mathrm{B}} \nabla C+D_{\mathrm{T}} \frac{\nabla T}{T_{\infty}}\right)$

where $\mathbf{V}(u(x, y), v(x, y), 0)$ is the velocity vector, $\rho$ is nanofluid density, $\mathbf{S}$ is Cauchy stress tensor, $\mathbf{b}$ is body force vector, $\rho c$ and $\rho_{\mathrm{p}} c_{\mathrm{p}}$ are heat capacities of nanofluid and nanoparticles, respectively, $T$ is temperature, $k$ is nanofluid thermal conductivity, $D_{\mathrm{B}}$ is Brownian diffusion coefficient, $C$ is nanoparticle volumetric fraction, $D_{\mathrm{T}}$ is thermophoretic diffusion coefficient and $T_{\infty}$ is the ambient fluid temperature. For Williamson fluid model Cauchy stress tensor $\mathbf{S}$ is defined in (Dapra 2007) as

$\mathbf{S}=-p \mathbf{I}+\tau$,

$\tau=\left[\mu_{\infty}+\frac{\left(\mu_{0}-\mu_{\infty}\right)}{1-\Gamma \dot{\gamma}}\right] \mathbf{A}_{1}$,

where $\tau$ is extra stress tensor, $\mu_{0}$ is limiting viscosity at zero shear rate and $\mu_{\infty}$ is limiting viscosity at infinite shear rate, $\Gamma>0$ is a time constant, $\mathbf{A}_{1}$ is the first Rivlin-Erickson tensor and $\dot{\gamma}$ is defined as follows:

$$
\begin{aligned}
\dot{\gamma} & =\sqrt{\frac{1}{2} \pi}, \\
\pi & =\operatorname{trace}\left(\mathbf{A}_{1}^{2}\right),
\end{aligned}
$$

Here we considered the case for which $\mu_{\infty}=0$ and $\Gamma \dot{\gamma}<1$. Thus Eq. (6) can be written as

$\tau=\left[\frac{\mu_{0}}{1-\Gamma \dot{\gamma}}\right] \mathbf{A}_{1}$,

or by using binomial expansion we get

$\tau=\mu_{0}[1+\Gamma \dot{\gamma}] \mathbf{A}_{1}$.

Making use of Eqs. (5) and (9) in Eqs. (1) to (4), the two-dimensional boundary layer equations governing the flow are given by

$\frac{\partial u}{\partial x}+\frac{\partial v}{\partial y}=0$,

$u \frac{\partial u}{\partial x}+v \frac{\partial u}{\partial y}=v \frac{\partial^{2} u}{\partial y^{2}}+\sqrt{2} v \Gamma \frac{\partial u}{\partial y} \frac{\partial^{2} u}{\partial y^{2}}$.

$u \frac{\partial T}{\partial x}+v \frac{\partial T}{\partial y}=\alpha \frac{\partial^{2} T}{\partial y^{2}}+\frac{\rho_{\mathrm{p}} c_{\mathrm{p}}}{\rho c}\left(D_{\mathrm{B}} \frac{\partial C}{\partial y} \frac{\partial T}{\partial y}+\frac{D_{\mathrm{T}}}{T_{\infty}}\left(\frac{\partial T}{\partial y}\right)^{2}\right)$,

$u \frac{\partial C}{\partial x}+v \frac{\partial C}{\partial y}=D_{\mathrm{B}} \frac{\partial^{2} C}{\partial y^{2}}+\frac{D_{\mathrm{T}}}{T_{\infty}} \frac{\partial^{2} T}{\partial y^{2}}$, 
where $u(x, y)$ and $v(x, y)$ are horizontal and vertical components of velocity, $v$ is kinematic viscosity and $\alpha$ is nanofluid thermal diffusivity. The corresponding boundary conditions are given by

$u=U_{\mathrm{w}} ; v=0 ; T=T_{\mathrm{w}}, C=C_{\mathrm{w}} \quad$ at $y=0$,

$u \rightarrow 0 ; T=T_{\infty}, C=C_{\infty} \quad$ as $y \rightarrow \infty$.

Since the surface is stretched with velocity $B x$, thus $U_{\mathrm{w}}=B x$. Introducing the following transformations in above equations

$u=B x f^{\prime}(\eta), \quad v=-\sqrt{B v} f(\eta), \quad \eta=\sqrt{\frac{B}{v}} y$

$\theta=\frac{T-T_{\infty}}{T_{\mathrm{w}}-T_{\infty}}, \quad \phi=\frac{C-C_{\infty}}{C_{\mathrm{w}}-C_{\infty}}$,

with the help of above transformations, Eq. (10) is identically satisfied and Eqs. (11) to (13) along with boundary conditions (14) take the following form:

$f^{\prime \prime \prime}-f^{\prime 2}+f f^{\prime \prime}+\lambda f^{\prime \prime} f^{\prime \prime \prime}=0$,

$\theta^{\prime \prime}+\operatorname{Pr} f \theta^{\prime}+\frac{N_{\mathrm{c}}}{L e} \phi^{\prime} \theta^{\prime}+\frac{N_{\mathrm{c}}}{L e \times N_{\mathrm{bt}}} \theta^{2}=0$,

$\phi^{\prime \prime}+S c f \phi^{\prime}+\frac{1}{N_{\mathrm{bt}}} \theta^{\prime \prime}=0$,

where $f, \theta$ and $\phi$ are functions of $\eta$ and prime denotes derivatives w.r.t $\eta$. The corresponding boundary conditions are

$f=0, f^{\prime}=1, \theta=1, \phi=1 \quad$ at $\eta=0$,

$f^{\prime}=0, \theta=0, \phi=0 \quad$ as $\eta \rightarrow \infty$.

In above transformed equations the following nondimensional parameters are introduced:

$\lambda=\Gamma x \sqrt{\frac{2 B^{3}}{v}}($ Non Newtonian Williamson parameter),

$\operatorname{Pr}=\frac{v}{\alpha}$ (Prandtl number $=$ momentum diffusivity $/$ nanofluid thermal diffusivity),

$L e=\frac{\alpha}{D_{\mathrm{B}}}$ (Lewis number $=$ nanofluid thermal diffusivity

/Brownian diffusivity),

$S c=\frac{v}{D_{\mathrm{B}}}($ Schmidt number $=$ momentum diffusivity

/Brownian diffusivity).

$N_{\mathrm{c}}=\frac{\rho_{\mathrm{p}} c_{\mathrm{p}}}{\rho c}\left(C_{\mathrm{w}}-C_{\infty}\right)($ Heat capacities ratio $=$ nano

particles heat capacity/nanofluid heat capacity),

$N_{\mathrm{bt}}=\frac{D_{\mathrm{B}} T_{\infty}\left(C_{\mathrm{w}}-C_{\infty}\right)}{D_{\mathrm{T}}\left(T_{\mathrm{w}}-T_{\infty}\right)}$ (Diffusivity ratio $=$ Brownian

diffusivity/thermophoretic diffusivity).
If we put $\lambda=0$, our problem reduces to the one for Newtonian nano and for $D_{\mathrm{B}}=D_{\mathrm{T}}=0$ in Eq. (12) our heat equation reduces to the classical boundary layer heat equation in the absence of viscous dissipation. Physical quantities of interest are Local skin friction coefficient $c_{\mathrm{f}}$, Local Nusselt number $N u$ and Local Sherwood number $S h$.

$$
\begin{aligned}
& c_{\mathrm{f}}=\frac{\tau_{\mathrm{w}}}{\rho U_{\mathrm{w}}^{2}}, \quad N u=\left.\frac{-x}{T_{\mathrm{w}}-T_{\infty}} \frac{\partial T}{\partial y}\right|_{y=0}, \\
& S h=\left.\frac{-x}{C_{\mathrm{w}}-C_{\infty}} \frac{\partial C}{\partial y}\right|_{y=0},
\end{aligned}
$$

or by introducing the transformations (15), we have

$$
\begin{aligned}
& \sqrt{\operatorname{Re}} c_{\mathrm{f}}=\left[\left(f^{\prime \prime}+\frac{\lambda}{2} f^{\prime \prime 2}\right)\right]_{\eta=0}, \frac{N u}{\sqrt{\operatorname{Re}}}=-\theta^{\prime}(0), \\
& \frac{S h}{\sqrt{\operatorname{Re}}}=-\phi^{\prime}(0)
\end{aligned}
$$

where $R e=B x^{2} / v$ is local Reynolds number. Physical parameters of interest will be discussed later in the results section.

\section{Solution technique}

Solutions of Eqs. (16-18) are obtained with the help of well-known Homotopy analysis technique (HAM). HAM is a strong analytic technique to solve linear and non-linear, ordinary and partial differential equations. HAM was developed by Liao in 1992 . HAM can be equally applied to weak and strong nonlinear problems because it is independent of small physical parameter restriction. It also provides a way to check and adjust the convergence of obtained solution with the help of auxiliary parameters and base functions. The initial guess and operators are taken as

$$
\begin{aligned}
f_{0}(\eta) & =1-\exp (-\eta), \\
\theta_{0}(\eta) & =\exp (-\eta), \\
\phi_{0}(\eta) & =\exp (-\eta), \\
L_{\mathrm{f}}(f) & =\frac{\mathrm{d}^{3} f}{\mathrm{~d} \eta^{3}}-\frac{\mathrm{d} f}{\mathrm{~d} \eta}, \\
L_{\theta}(\theta) & =\frac{\mathrm{d}^{2} \theta}{\mathrm{d} \eta^{2}}+\frac{\mathrm{d} \theta}{\mathrm{d} \eta}, \\
L_{\phi}(\phi) & =\frac{\mathrm{d}^{2} \phi}{d \eta^{2}}+\frac{\mathrm{d} \phi}{\mathrm{d} \eta} .
\end{aligned}
$$

The convergence of Homotopy analysis solution greatly depends on the choice of auxiliary parameter $h_{\mathrm{f}}, h_{\theta}, h_{\phi}$.

\section{Results and discussion}

In order to check the convergence of obtained solutions, combine $h$-curve is plotted. It can be observed from Fig. 1 


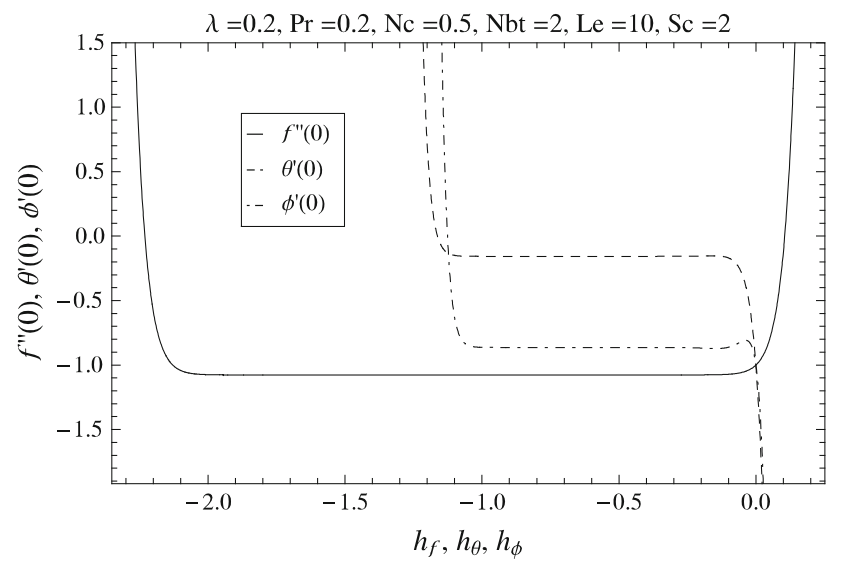

Fig. 1 Combine plot for $h$ curves

Table 1 Convergence of HAM solution for different order of approximation when $\operatorname{Pr}=0.5, N_{\mathrm{c}}=0.5, N_{\mathrm{bt}}=2, L e=4$, $S c=2, \lambda=0.2$ and $h=h_{\mathrm{f}}=h_{\theta}=h_{\phi}=-0.7$

\begin{tabular}{llll}
\hline Order of approximation & $-f^{\prime \prime}(0)$ & $-\theta^{\prime}(0)$ & $-\phi^{\prime}(0)$ \\
\hline 1 & 1.047 & 0.409 & 0.650 \\
5 & 1.076 & 0.302 & 0.835 \\
10 & 1.076 & 0.308 & 0.824 \\
18 & 1.076 & 0.308 & 0.824 \\
25 & 1.076 & 0.308 & 0.824 \\
30 & 1.076 & 0.308 & 0.824 \\
\hline
\end{tabular}

that the admissible range for $h_{\mathrm{f}}, h_{\theta}, h_{\phi}$ is $-1.9 \leq h_{\mathrm{f}} \leq$ $-0.3,-0.9 \leq h_{\theta} \leq-0.3,-1.0 \leq h_{\phi} \leq-0.3$. Convergence table has been drawn for $f^{\prime \prime}(0), \phi^{\prime}(0), \theta^{\prime}(0)$ when $h=h_{\mathrm{f}}=h_{\theta}=h_{\phi}=-0.7$. It is found that the convergence is achieved at 10th order of approximation (Table 1). All the tables and graphs are plotted at 22nd order of approximation.

From Fig. 2 it is observed that for a nanofluid the velocity as well as the boundary layer thickness decreases with the increase in non-Newtonian parameter $\lambda$. With increase in Prandtl number $\mathrm{Pr}$, temperature as well as thermal boundary layer thickness decreases, see Fig. 3. It is also noted that the temperature and thermal boundary layer thickness decrease with increase in lewis number $L e$ and $N_{\mathrm{bt}}$ (Figs. 4, 5). Since $N_{\mathrm{bt}}$ is the ratio of Brownian to thermophoretic diffusivities, increase in $N_{\mathrm{bt}}$ means greater activity of nanofluid particles. While they increase with increase in $\lambda$ and $N_{\mathrm{c}}$ (see Figs. 6, 7). To observe the effects of different parameters on nanoparticle volume fraction, graphs have been plotted against $\lambda, S c$ and $N_{\mathrm{bt}}$. Nanoparticle volume fraction decreases with increase in Schmidt number $S c$ and $N_{\mathrm{bt}}$, while it decrease with increase in $\lambda$. (See Figs. 8, 9, 10). It can also be observed from Fig. 11 that nanoparticle volume fraction (close to the boundary)

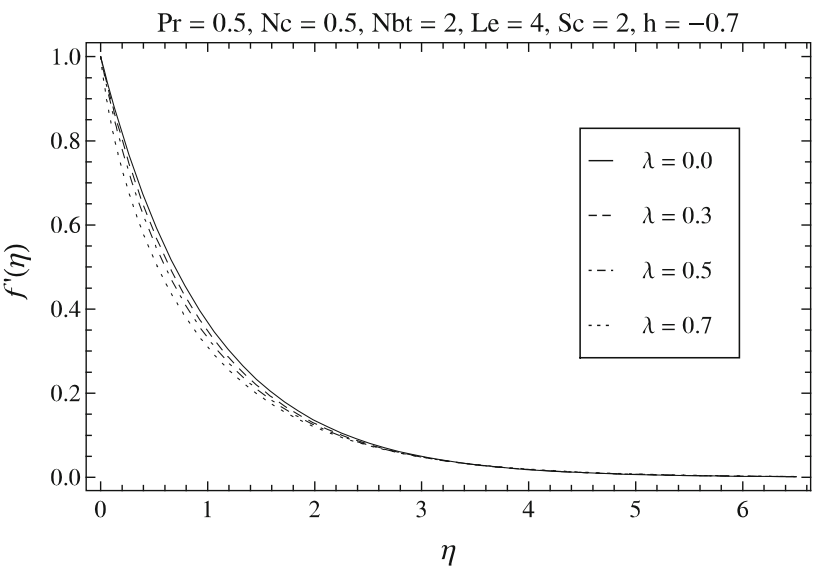

Fig. 2 Velocity variation against different values of $\lambda$

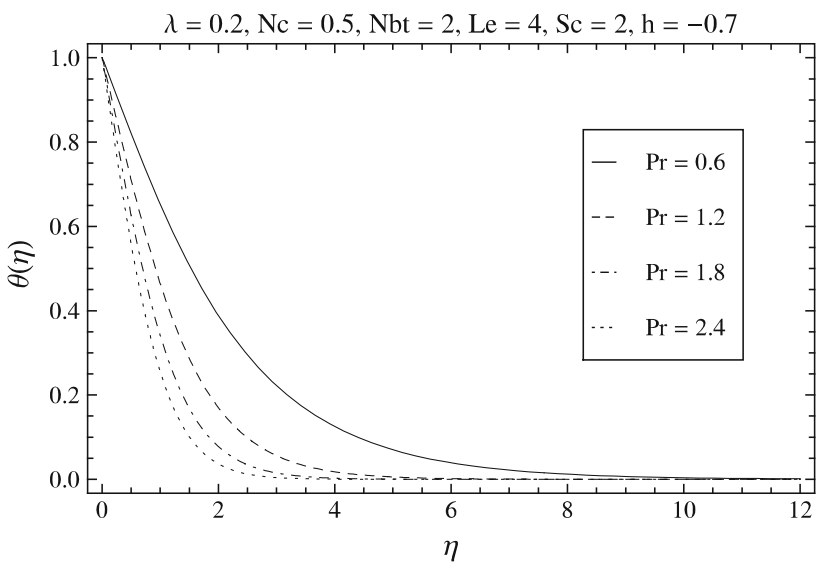

Fig. 3 Temperature variation against different values of $\mathrm{Pr}$

increases with increase in $P r$. The behaviour shift of graph away from wall greatly depends on the values of $N_{\mathrm{bt}}$. Since Eq. (18) is a second-order differential equation in both $\theta$ and $\phi$, here the only thing which can make difference is the value of $N_{\mathrm{bt}}$. Also if the value of $N_{\mathrm{bt}}$ is very large then $\phi$ hardly depends on $\theta$. Therefore, effects of Prandtl number almost diminish for very large values of $N_{\mathrm{bt}}$. It can also be observed from the graphs of velocity, temperature and Nanoparticle volume fraction that nanoparticle volume fraction and temperature boundary layers survive longer as compared with the velocity boundary layer. The importance of nanofluid study is because of heat transfer enhancement. To observe the effects of effective parameters on heat transfer closed to the wall, we plotted the graphs for $-\theta^{\prime}(\eta)$. It can be observed from Figs. 12 and 13 that the heat transfer in fluid increases with the increase in $N_{\text {bt }}$ and $L e$; this enhancement is very significant in the region very close to the wall but the parameter effect is almost negligible on heat transfer away from the wall. Table 2 is drawn to compare our results for the viscous case in the absence of nanoparticles. These results are 


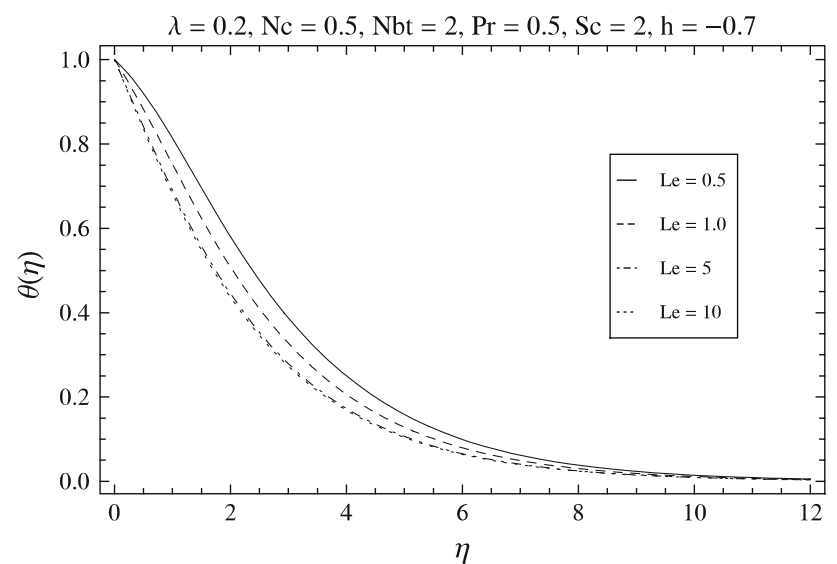

Fig. 4 Temperature variation against different values of $L e$

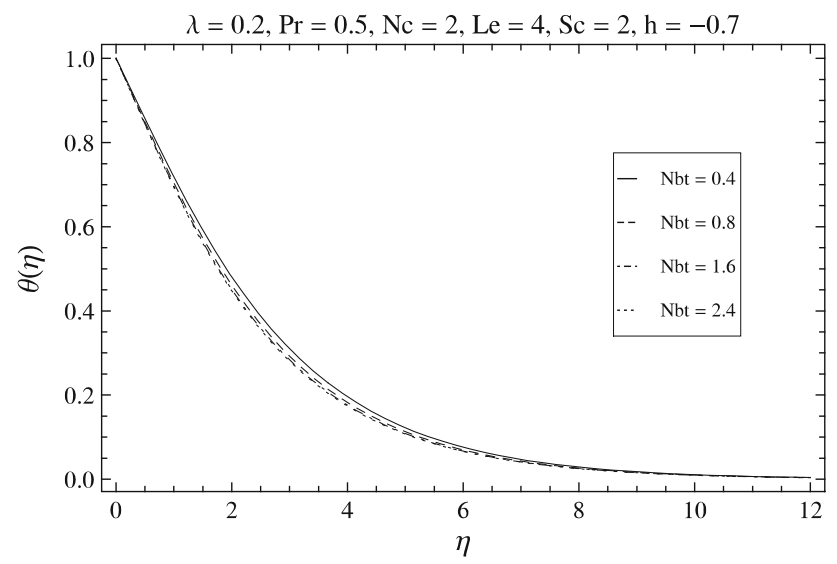

Fig. 5 Temperature graph for different values of $N_{\mathrm{bt}}$

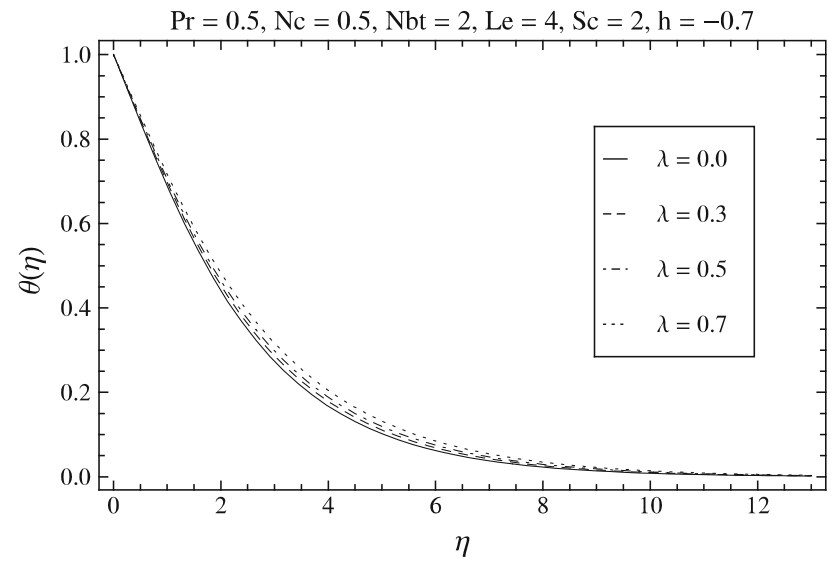

Fig. 6 Temperature variation against different values of $\lambda$

found to be in good agreement. Tables 3 and 4 show wall temperature gradient and wall nanoparticle volume fraction gradient respectively. It is observed that the wall temperature gradient decreases with the increase in $\lambda$ and $N_{\mathrm{c}}$. Here $L e$ and $N_{\mathrm{bt}}$ are important heat transfer parameters for nanofluids; it is observed that the heat transfer increases

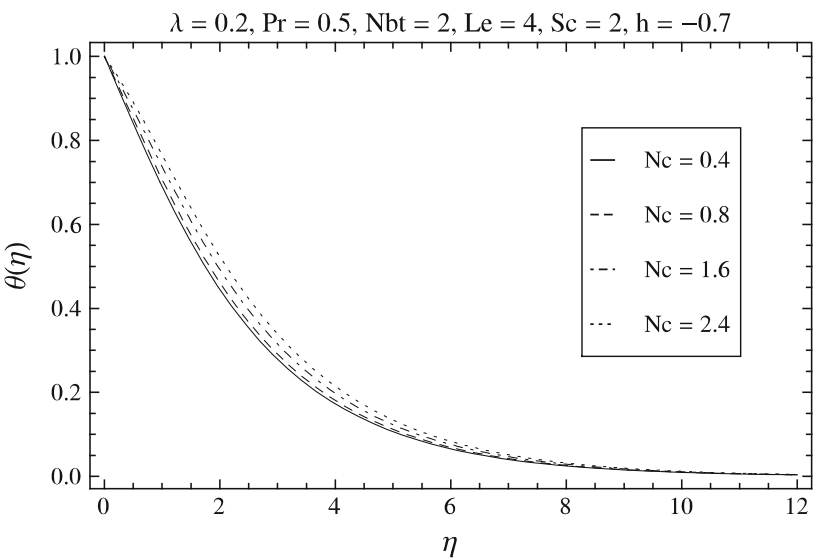

Fig. 7 Temperature variation against different values of $N_{\mathrm{c}}$

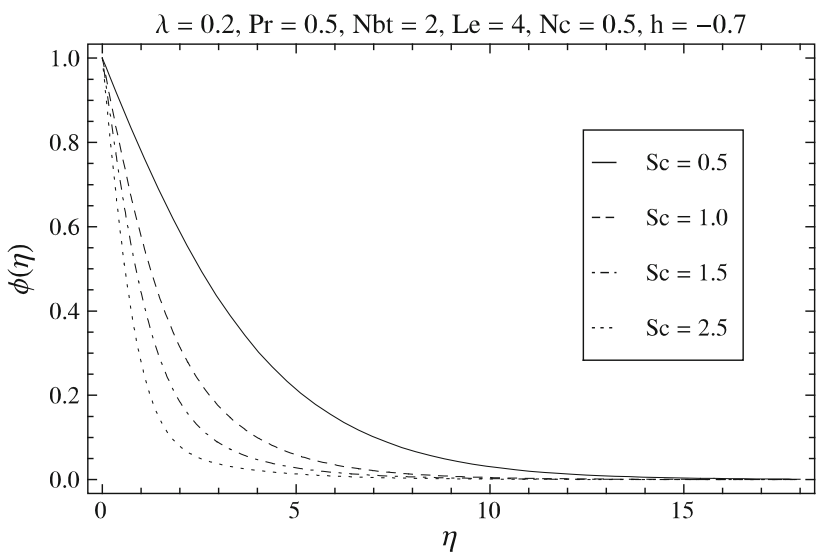

Fig. 8 Nanoparticles volume fraction variation against different values of $S c$

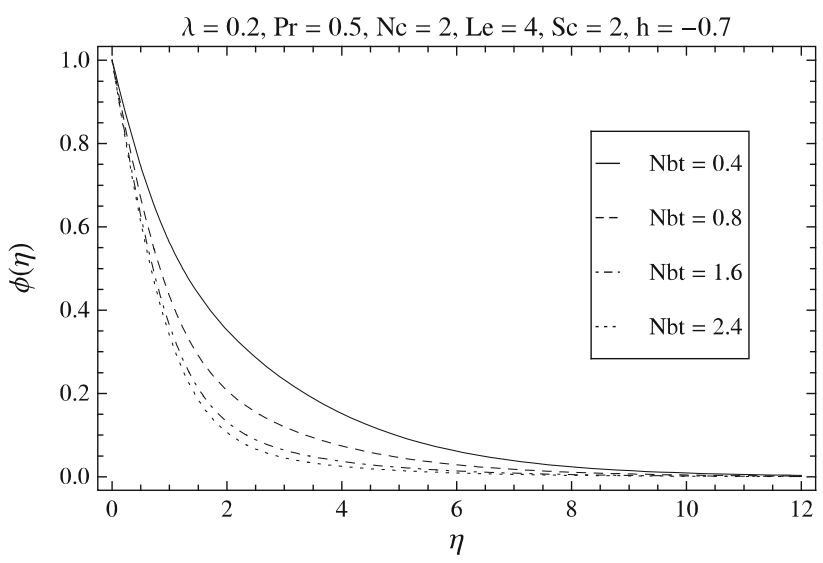

Fig. 9 Nanoparticle volume fraction against different values of $N_{\mathrm{bt}}$

with the increase in both parameters. Also wall temperature gradient increases with the increase in Prandtl number. From Table 4, it can be observed that wall nano particle volume fraction gradient decreases with the increase in $\lambda$ and $L e$ while it increases with the increase in $N_{\mathrm{bt}}$ and $S c$. 


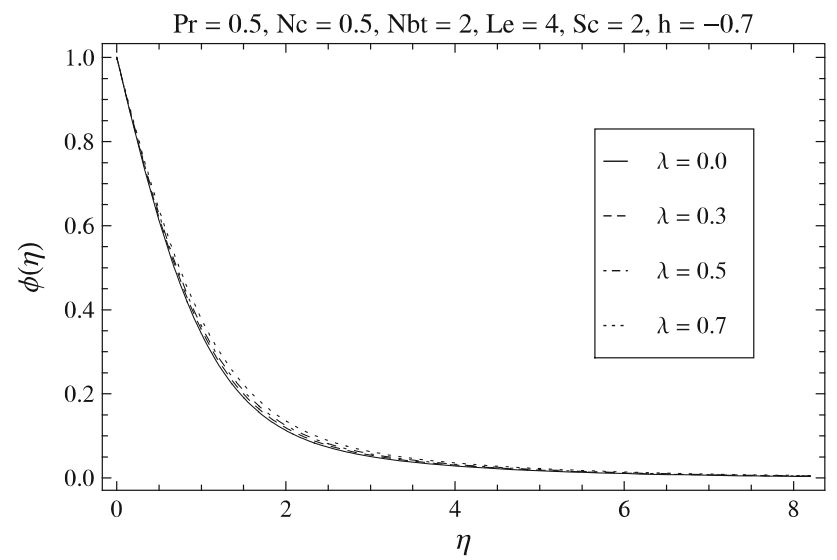

Fig. 10 Nanoparticle volume fraction variation against different values of $\lambda$

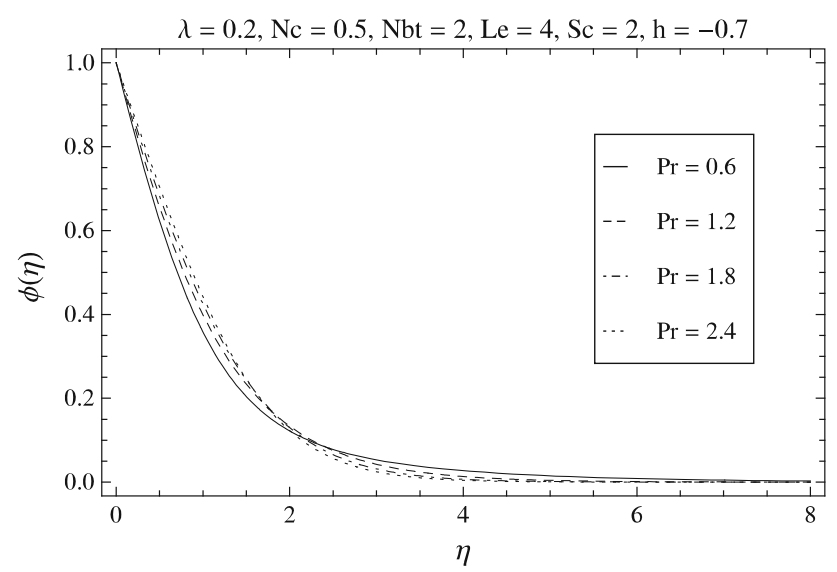

Fig. 11 Nanoparticle volume fraction against different values of $\mathrm{Pr}$

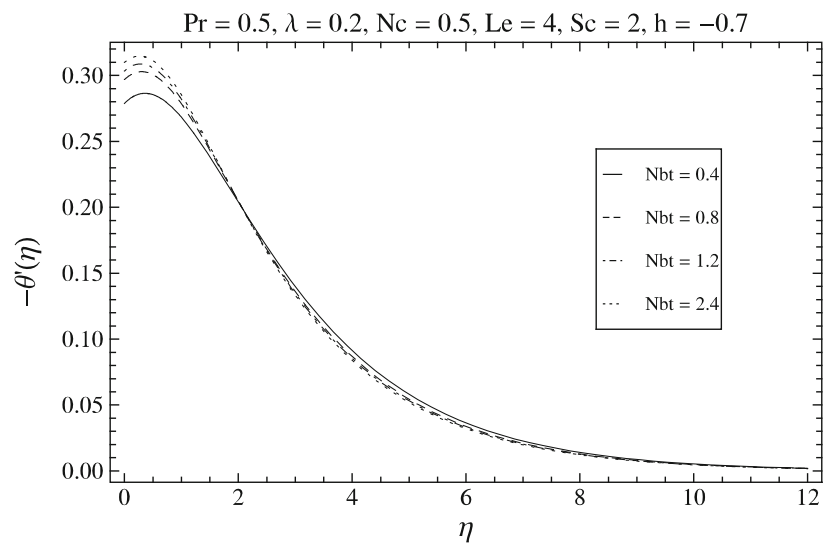

Fig. 12 Heat transfer in fluid against different values of $N_{\mathrm{bt}}$

\section{Concluding remarks}

In the present paper we tried to analyse the nano particle effect on boundary layer flow of Williamson fluid over a

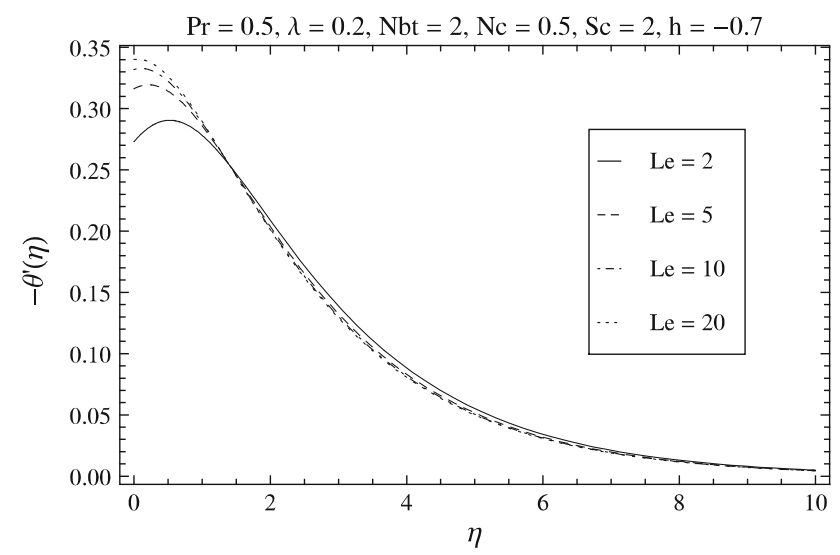

Fig. 13 Heat transfer in fluid against different values of $L e$

Table 2 Comparison table for viscous case $\left(-\theta^{\prime}(0)\right)$

\begin{tabular}{lllll}
\hline$P r$ & $\begin{array}{l}\text { Present } \\
\text { results }\end{array}$ & $\begin{array}{l}\text { Khan and Pop } \\
(2010)\end{array}$ & $\begin{array}{l}\text { Wang } \\
(1989)\end{array}$ & $\begin{array}{l}\text { Golra and Sidawi } \\
(1994)\end{array}$ \\
\hline 0.07 & 0.066 & 0.066 & 0.066 & 0.066 \\
0.20 & 0.169 & 0.169 & 0.169 & 0.169 \\
0.70 & 0.454 & 0.454 & 0.454 & 0.454 \\
2.0 & 0.911 & 0.911 & 0.911 & 0.911 \\
\hline
\end{tabular}

Table 3 Values of wall temperature gradient $-\theta^{\prime}(0)$, when $h=-0.7$

\begin{tabular}{llllll}
\hline$\lambda$ & $L e$ & $N_{\mathrm{bt}}$ & $N_{\mathrm{c}}$ & $P r$ & $-\theta^{\prime}(0)$ \\
\hline 0.0 & 4.0 & 2.0 & 0.5 & 0.5 & 0.314 \\
0.2 & & & & & 0.309 \\
0.4 & & & & & 0.302 \\
0.2 & 4.0 & 2.0 & 0.5 & 0.5 & 0.309 \\
& 10 & & & & 0.332 \\
& 20 & & & & 0.340 \\
0.2 & 4.0 & 0.5 & 0.5 & 0.5 & 0.286 \\
& & 1.0 & & & 0.301 \\
& & 2.0 & & & 0.309 \\
0.2 & 4.0 & 2.0 & 0.5 & 0.5 & 0.309 \\
& & & 1.0 & & 0.273 \\
& & & 2.0 & & 0.213 \\
0.2 & 4.0 & 2.0 & 0.5 & 0.2 & 0.144 \\
& & & & 0.6 & 0.355 \\
& & & & 1.2 & 0.588 \\
\hline
\end{tabular}

stretching surface. The governing non-linear equations are solved analytically using HAM. The important findings of the paper are as follows:

- Lewis number will appear in nano particle volume fraction equation for the case when velocity 
Table 4 Values of wall nano particle volume fraction gradient $-\phi^{\prime}(0)$, when $h=-0.7$

\begin{tabular}{lllll}
\hline$\lambda$ & $L e$ & $N_{\mathrm{bt}}$ & $S c$ & $-\phi^{\prime}(0)$ \\
\hline 0.0 & 4.0 & 2.0 & 0.5 & 0.833 \\
0.2 & & & 0.824 \\
0.4 & & & 0.811 \\
0.2 & 4.0 & 2.0 & 0.5 & 0.824 \\
& 10 & & & 0.815 \\
& 20 & & & 0.812 \\
0.2 & 4.0 & 0.5 & 0.5 & 0.620 \\
& & 1.0 & & 0.751 \\
& & 2.0 & & 0.823 \\
0.2 & 4.0 & 2.0 & 0.5 & 0.228 \\
& & & 1.0 & 0.475 \\
& & & 2.0 & 0.824 \\
\hline
\end{tabular}

transformation depends on thermal diffusivity; otherwise, Schmidt number will appear.

- The parameters have strong impact on heat transfer very close to the wall and are almost negligible slightly away from wall.

- Wall temperature gradient increases with increase in $\mathrm{Le}$ and $N_{\mathrm{bt}}$.

- Wall nano particle fraction gradient increases with $N_{\mathrm{bt}}$ and $S c$ and decreases with $L e$.

Open Access This article is distributed under the terms of the Creative Commons Attribution License which permits any use, distribution, and reproduction in any medium, provided the original author(s) and the source are credited.

\section{References}

Abbasbandy S (2006) The application of homotopy analysis method to nonlinear equations arising in heat transfer. Phys Lett A 360(1):109-113

Abbasbandy S (2007) The application of homotopy analysis method to solve a generalized Hirota-Satsuma coupled $\mathrm{KdV}$ equation. Phys Lett A 361(6):478-483

Ali ME (1995) On thermal boundary layer on a power law stretched surface with suction or injection. Int $\mathbf{J}$ Heat Mass Transf 16:280-290

Bachok N, Ishak A, Pop I (2010) Boundary-layer flow of nanofluids over a moving surface in a flowing fluid. Int $\mathrm{J}$ Therm Sci 49:1663-1668

Buongiorno J (2006) Convective transport in nanofluids. J Heat Transf 128:240-250

Choi SUS (1995), Enhancing thermal conductivity of fluids with nanoparticles. In: The proceedings of the 1995 ASME International Mechanical Engineering Congress and Exposition. ASME, San Francisco, USA, FED 231/MD 66, pp 99-105

Dapra, Scarpi G (2007) Perturbation solution for pulsatile flow of a non-Newtonian Williamson fluid in a rock fracture. Int J Rock Mech Min Sci 44:271-278

Durmus, Kasgoz A, Macosko CW (2007) Linear low density polyethylene (LLDPE)/clay nanocomposites. Part I: structural characterization and quantifying clay dispersion by melt rheology. Polymer 48:4492-4502

Ellahi R, Riaz A (2010) Analytical solutions for MHD flow in a thirdgrade fluid with variable viscosity. Math Comput Model 52(9-10): 1783-1793

Erickson LE, Fan LT, Fox VG (1966) Heat and mass transfer in the laminar boundary layer flow of a moving flat surface with constant surface velocity and temperature focusing on the effects of suction/injection. Ind Eng Chem Fundam 5:19-25

Gorla RSR, Sidawi I (1994) Free convection on a vertical stretching surface with suction and blowing. Appl Sci Res $52: 247-257$

Hayat T, Qasim M (2010) Influence of thermal radiation and Joule heating on MHD flow of a Maxwell fluid in the presence of thermophoresis. Int $\mathbf{J}$ Heat Mass Transf 53(21-22): $4780-4788$

Kandasamya R, Loganathanb P, Puvi Arasub P (2011) Scaling group transformation for MHD boundary-layer flow of a nanofluid past a vertical stretching surface in the presence of suction/injection. Nucl Eng Des 241:2053-2059

Kelson NA, Farrell TW (2001) Micropolar flow over a porous stretching sheet with strong suction or injection. Int Commun Heat Mass Transf 28(4):479-488

Khan WA, Pop I (2010) Boundary-layer flow of a nanofluid past a stretching sheet. Int J Heat Mass Transf 53:2477-2483

Kumaran V, Ramanaiah G (1996) A note on the flow over a stretching sheet. Acta Mech 116(1-4):229-233

Liao S (2003) Beyond perturbation: introduction to the homotopy analysis method. Chapman and Hall/CRC, Boca Raton, pp 99-102

Liao S (2004) On the homotopy analysis method for nonlinear problems. Appl Math Comput 147:499-513

Liu C (2005) A note on heat and mass transfer for a hydromagnetic flow over a stretching sheet. Int Commun Heat Mass Transf 32(8):1075-1084

Lyubimov V, Perminov AV (2002) Motion of a thin oblique layer of a pseudoplastic fluid. J Eng Phys Thermophys 75(4):920-924

Makinde OD, Aziz A (2011) Boundary layer flow of a nanofluid past a stretching sheet with a convective boundary condition. Int $\mathbf{J}$ Therm Sci 50:1326-1332

Masuda H, Ebata A, Teramae K, Hishinuma N (1993) Alteration of thermal conductivity and viscosity of liquid by dispersing ultrafine particles. Netsu Bussei 7(4):227-233

Nadeem S, Akbar NS (2010) Numerical solutions of peristaltic flow of Williamson fluid with radially varying MHD in an endoscope. Int J Numer Methods Fluids 66(2):212-220

Nadeem S, Hussain A (2009) MHD flow of a viscous fluid on a nonlinear porous shrinking sheet with homotopy analysis method. Appl Math Mech 30(12):1569-1578

Nadeem S, Hussain A, Vajravelu K (2010) Effects of heat transfer on the stagnation flow of a third order fluid over a shrinking sheet. Zeitschrift für Naturforschung 65(a):969-994

Nadeem S, Hussain ST, Lee C (2013) Flow of a Williamson fluid over a stretching sheet. Braz J Chem Eng 30(3):619-625

Nield DA, Kuznetsov AV (2009) Thermal instability in a porous medium layer saturated by a nanofluid. Int $\mathrm{J}$ Heat Mass Transf 52:5796-5801

Nield DA, Kuznetsov AV (2011) The onset of double-diffusive convection in a nanofluid layer. Int $\mathbf{J}$ Heat Fluid Flow 32:771-776

Rosali H, Ishak A, Pop I (2012) Micropolar fluid flow towards a stretching/shrinking sheet in a porous medium with suction. Int Commun Heat Mass Transf 39(6):826-829

Sakiadis C (1961) Boundary layer behavior on continuous solid flat surfaces. Am Inst Chem Eng J 7:26-28 
Tsou FK, Sparrow EM, Goldstein RJ (1967) Flow and heat transfer in the boundary layer on a continuous moving surface. Int J Heat Mass Transf 10:219-235

Wang Y (1989) Free convection on a vertical stretching surface. J Appl Math Mech 69:418-420
Williamson RV (1929) The flow of pseudoplastic materials. Ind Eng Chem 21(11):1108-1111 